\title{
Exploring Lifelog Sharing and Privacy
}

Soumyadeb Chowdhury

Singapore Institute of Technology

10 Dover Drive, Singapore,

Soum.Chowdhury@singaporetech.edu.sg

\section{Md Sadek Ferdous}

University of Southampton

Southampton SO17 1BJ, UK

S.Ferdous@soton.ac.uk

Joemon M Jose

University of Glasgow

Glasgow G12 8QQ, UK

Joemon.Jose@glasgow.ac.uk
Permission to make digital or hard copies of part or all of this work for personal or classroom use is granted without fee provided that copies are not made or distributed for profit or commercial advantage and that copies bear this notice and the full citation on the first page. Copyrights for third-party components of this work must be honored. For all other uses, contact the Owner/Author.

uses, contact the Owner/Author.
Copyright is held by the owner/author(s).

Ubicomp/ISWC'16 Adjunct, September 12-16, 2016, Heidelberg, Germany

Germany

http://dx.doi.org/10.1145/2968219.2968320

\begin{abstract}
The emphasis on exhaustive passive capturing of images using wearable cameras like Autographer, which is often known as lifelogging has brought into foreground the challenge of preserving privacy, in addition to presenting the vast amount of images in a meaningful way. In this paper, we present a user-study to understand the importance of an array of factors that are likely to influence the lifeloggers to share their lifelog images in their online circle. The findings are a step forward in the emerging area intersecting HCI, and privacy, to help in exploring design directions for privacy mediating techniques in lifelogging applications.
\end{abstract}

\section{Author Keywords}

Lifelogging; HCI; Privacy; Wearable Camera

\section{ACM Classification Keywords}

K.4.1. Public Policy Issues: Privacy; K.4.2. Social Issues

\section{Introduction}

Lifelogging uses modern digital technologies like wearable cameras (Figure 1 ) to record entire lives of a lifelogger ( $L L$, the person wearing the device) in a series of passively captured images. However, it is important to note that in addition to recording their own lives, LLs are recording others (often referred to as bystanders - BYs, known or unknown people captured in the photographs), as well. The images may be captured in different settings/scenarios including, but 


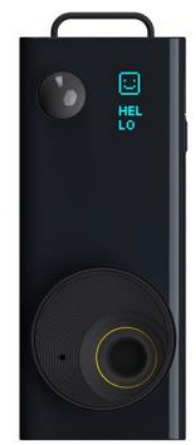

Figure 1: Autographer (Wearable Camera)

\section{Q1 Responses}

1

Embarrassing moments

2

Content hurting sentiments

3

Images of family members

4

Who can view the images

5 Images of tragic events

Images captured in

6 confidential meetings, nightclubs etc.

Table 1: Responses for Q1 images won't share online not limited to private (living room), intimate (time spent with love ones), corporate (workplace). We believe that as lifelogging cameras become mainstream, LLs are likely to share these images in their online social circle for myriad reasons. In this context, the findings reported in [3] have suggested that sharing lifelog images will motivate wearing such devices, and eventually make it popular. The practice of sharing lifelog images is likely to impact the privacy of both the LLs and BYs. Given the vast number of images captured by the wearable cameras, it is necessary to design applications that will strike a balance between preserving the privacy of the LLs and BYs, without undermining the user experience. In this paper, we present a user study which attempts to understand the importance of an array of factors (derived from the literature) from the LLs' perspective that will influence their decision to share lifelog images online. These findings can be considered as a starting point to create a privacy framework (which ought to be enhanced in the future) that can automatically recommend the suitability of an image to be shared online.

\section{Literature Survey}

Privacy is a key aspect of the user experience with new technologies [1]. However, the users' experience, expectations, and issues concerning privacy are most likely to differ when moving among areas of computing, society and even tasks. Given the diversity of users' views in relation to privacy, which is extremely contextual, there is a need to understand their perceptions and attitudes by conducting empirical studies, which will help to develop user friendly applications, addressing their privacy concerns. The literature on lifelogging privacy is sparse, and has not reported human subject experiments except [8 and 9] to understand privacy implications from the perspectives of the LLs. Zhou and Gurrin [12] have identified privacy as one of the primary concerns of the LLs. Gürses et al. [10] have argued that privacy by design lacks details as to how it can be implemented, while meeting the requirements of a system. Hoyle et al. [8] have suggested that sensitivity of an image can be determined by the combination of factors which include time, location, objects and people appearing in the image. Hoyle et al. [9] have also argued that LLs respect the privacy of the BSs. However, they have not differentiated between people known or unknown to the LLs. We contend that the LLs' concern about a BS's privacy is likely to depend upon the relationship they share with the bystander. For example, it is highly unlikely that the concern of a LL for their family members will be same as their colleagues in workplace or a stranger in the crowd. In this paper, we seek to explore how the importance of a number of factors influencing image sharing may differ to share lifelog images, from the LLs' point of view, facilitating the development of privacy preserving mechanisms for such an emerging ubiquitous technology.

\section{User Study}

A user-study comprising of three stages was conducted using the within-subjects design. 15 postgraduate students in Glasgow (Female: 5; Male: 10; age range: 21-30) voluntarily took part in our study. None of them had used such devices in the past, which was confirmed through the pre-study questionnaire. The first stage (S1) had two phases (S1P1 and S1P2). The questions that were asked in each phase are presented in Table 2 and 3. The responses to the question (Q1) asked in S1P1 were analyzed (Table 1 ) in addition to the literature reported in [8] to derive a number of factors 
id

\section{Questions}

Q1

Image they don't like

to share online

Q2

Reasons for

capturing images

Reasons for

Q3

reviewing images (if

applicable)

Q4

Types of images

deleted

Rate factors that may influence the decision to share images in the online social circle

How did the device affect the lifestyle

Table 2: List of questions asked during the user-study. Questions corresponding to each stage are reported in Table 3

\begin{tabular}{l|l} 
id & Stage(s) \\
\hline Q1 & S1P1 \\
Q2 & S1P2, S2, S3 \\
Q3 & S3 \\
Q4 & S3 \\
Q5 & S1P2, S2, S3 \\
6 & $\mathrm{~S} 3$
\end{tabular}

Table 3: Questions asked in each stage of the user study that are likely to affect sharing decisions of the LLs (i.e. factors that LLs were required to rate in Q5), presented in Table 5. S1P2 was conducted 4 days after S1P1, where LLs answered questions (Q2 and Q5). The second stage (S2) was conducted a week after S1P2, and LLs were not required to do any task during this gap. LLs were provided with a 50 word textual information about the characteristics of the Autographer, and then asked to answer a number of questions to verify that they understand the characteristics of the device. All the LLs answered these questions correctly. We hypothesized that the responses to these questions in $\mathrm{S} 2$ will be similar to $\mathrm{S} 1$, which is likely attributed to the tendency of the users to follow the same habits they are used to in their daily life. This stage is likely to reflect the perceptions of users who have read about such devices over the web, but have not used the device. Finally in the third stage (S3), a week after completing S2, the LLs were given two devices: Autographer and GPS tracker (recording location logs every 5 seconds). They were also provided with an information sheet to help them use the devices, in addition to a demonstration. The devices were allocated during the start of the week (i.e. Monday), and the LLs were asked to use them for 2-3 days. The time frame to collect the lifelogs was limited due to the voluntary nature of participation, and reduce subject attrition. The devices were returned on Friday in person, when the LLs were also asked to use our lifelog web application and complete the following tasks: (1) upload the images and GPS logs; (2) optional choice to review their images, and delete as applicable; (3) view the uploaded images and GPS logs in application. Semi-structured interviews were also conducted in S3. Unlike Hoyle et al. [8], instead of a single stage study, we chose to conduct a 3 stage study to explore how the perspectives of the LLs are likely to evolve or change from the control stage (S1) to the final stage (S3), and understand, whether privacy frameworks and design decisions for the lifelogging applications are likely to be different from the existing image sharing services. This study was approved by the ethics committee of our institution. A list of suggestions were provided to the LLs in order to reduce the potential risks arising from our study. The suggestions included, but were not limited to: (1) avoiding using the device in rest rooms and in places where photography may be prohibited; (2) if a BY enquires about the device, first the image capture must be paused, and then the objectives of our study must be explained; (3) if a BY seems concerned, then follow step 2, provide them with our contact information, and take a note of the date and time of the incident in a deletion card, so that we were able to delete the images captured during that period of time.

\section{Results}

Reasons for capturing images

The responses for each stage revealed that the popular reasons (10 out of 15 responses) for capturing images are similar, but not limited to: capturing important moments and sharing these memories with their family and friends; keeping in touch with their online social circle and sharing with them the daily activities. Additionally, a number of responses in S3 echoed sharing one distinct image for a period of ' $n$ ' days, which is increasingly becoming popular in Facebook.

Reasons for reviewing and deleting images

A total of 13,725 images were captured by the 15 LLs. One $L L$ refused to upload and store the images captured (1071 in total) in our server, due to personal reasons. The statistics related to the data capture is 


\begin{tabular}{l|l}
\multicolumn{1}{c}{ Images } & \multicolumn{1}{c}{$\#$} \\
\hline captured & 13725 \\
Uploaded & 12654 \\
Deleted & 1485 \\
Stored & 11169 \\
Duplicates & 6914 \\
Blurred & 3775 \\
Locations & 2990
\end{tabular}

Table 4: Lifelog image capture statistics

\begin{tabular}{c|l}
\multicolumn{1}{c}{ id } & \multicolumn{1}{|c}{ Factors } \\
\hline F1 & Content [8] \\
F2 & Scenario/settings (Q1) \\
F3 & Sensitivity (Q1) \\
F4 & Known people (Q1) \\
F5 & Unknown people (Q1) \\
F6 & Audience (Q1) \\
F7 & Mocking/ making fun \\
& (Q1) \\
F8 & Impression [8] \\
F9 & Tracking [8] \\
F10 & Location [8]
\end{tabular}

Table 5: Factors influencing image share presented in Table 4. During S3, all the $14 \mathrm{LLS}$ (excluding the individual who refused to upload), did not skip the option to review the images. The popular reasons (10 out of 14 responses in total) to review their lifelogs were, but not limited to: (1) the camera was used in various scenarios, so they would like to discard their private images; (2) they do not want to store the images which have captured either their close ones or themselves in potentially intimate or embarrassing moments; (3) they are likely to store the contents, which seem interesting to share. We found that the mean time spent in reviewing and deleting the images is 9.53 mins (SD: $2.55, \mathrm{SE}: 0.68$ ). Out of 12654 images uploaded, the LLs deleted 1485 images (Mean: 99, SD: 22.88, SE: 6.11). 10 LLs deleted more than 100 images. The popular reasons (10 responses out of 15) for deleting the images are: objects in the image; image scenarios/settings; known people in the image: private moments; self-concern; image location;

ATM/computer screen. The LLs reported that the review process was time-consuming, and efforts should be made to make it efficient.

Factors influencing image Share

The LLs were asked to rate the importance of a number of factors (Table 5 ) on a 5 point scale ( 1 being the least important and 5 being the most important) that are likely to determine the suitability of lifelog images to be shared in their online social circle. The decreasing order of importance of factors in 51 (Figure 2) based upon the mean score (Ms) is: Sensitivity (4.47) $>$ Scenario (4.40) $>$ Content $=$ Audience (4.1) $>$ Impression (4) > Known people (3.6) $>$ Tracking (3.36) $>$ Mocking (3.12) > Location (2.78) > Unknown people (2.53). We also found that the mean scores for all the factors in S2 followed similar trends as that of $S 1$, without any significant differences $(p>0.05)$. During $S 3$, the factors - tracking, known people and location, were rated important (Ms > 4), in addition to the five factors ( $F 1$, $\mathrm{F} 2, \mathrm{~F} 3, \mathrm{~F} 6$ and F8) in S1, and were statistically significant $(p=0.001)$, compared to the preceding stages. The responses in S3 showed that: (1) LLs are less concerned about the privacy of the unknown people (BYs) compared to known people, which is also statistically significant $(p=0.018) ;(2)$ the perceived importance of the factors differed in the case of lifelog images compared to images captured actively by the LLs using their personal hand-held devices. We acknowledge that there is often a gap between people's stated preferences, and views, since the privacy sphere is relative. Hence the results are likely to differ for users across different age groups, and perhaps countries. However, these findings can be considered as a step forward to understand perspectives of the LLs, which will help us to design privacy friendly lifelogging applications.

LifeLogging affecting lifestyle

The semi-structured interviews conducted in S3 revealed that the LLs had to remember to pause or put the device away, while reviewing confidential information (ATMs), and using facilities, which was a bit annoying. The LLS reported that they were required to pay more attention to the tasks involved in the study rather than focusing on the activities in their daily life. These results showed that the LLs became vigilant and anxious towards lifelogging more than concentrating on their day to day activities in life. 


\begin{tabular}{|c|c|}
\hline id & Factors \\
\hline $\mathrm{F} 1$ & Objects in the image \\
\hline $\mathrm{F} 2$ & $\begin{array}{l}\text { Formal and informal } \\
\text { gathering }\end{array}$ \\
\hline F3 & Confidential \\
\hline F4 & Family, friends. \\
\hline F5 & General public \\
\hline F6 & Who can view \\
\hline F7 & $\begin{array}{l}\text { Image likely to hurt } \\
\text { someone's sentiment }\end{array}$ \\
\hline F8 & $\begin{array}{l}\text { Way image portrays } \\
\text { LL/ known associates }\end{array}$ \\
\hline F9 & $\begin{array}{l}\text { If the activities can } \\
\text { be tracked }\end{array}$ \\
\hline F10 & $\begin{array}{l}\text { Where the image is } \\
\text { captured, common } \\
\text { space, rest room, } \\
\text { workplace etc. }\end{array}$ \\
\hline
\end{tabular}

Table 6: Brief description of each factor mentioned in Table 4

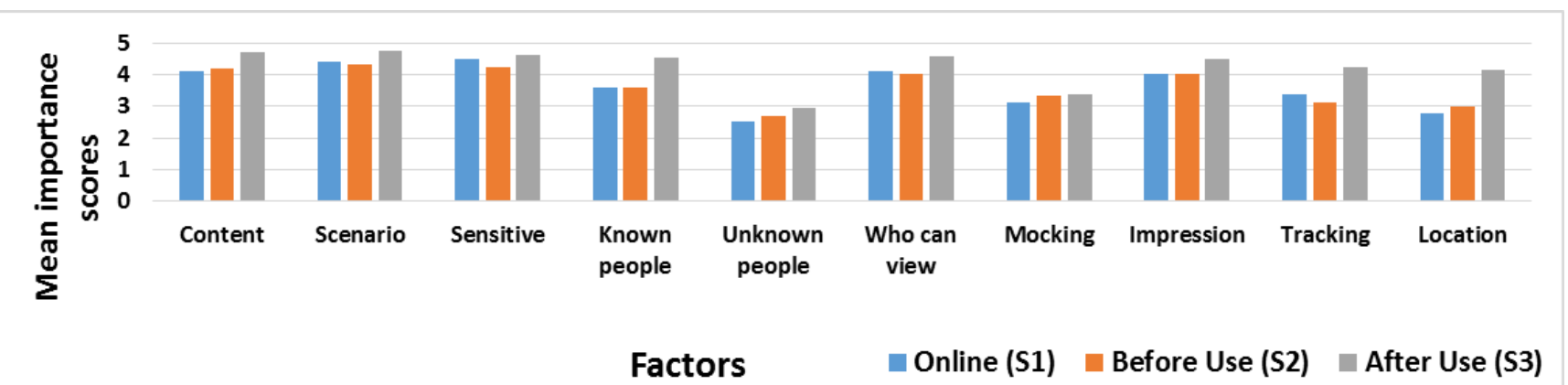

Figure 2: Factors determining the suitability of images to be shared online.

\section{Conclusion}

The results demonstrated that LLs are likely to share lifelog images in their social circle, review their lifelogs, and delete the ones that they perceive as sensitive, private, and uninteresting.. Based on our findings, we contend that lifelogging is not necessarily limited to personal informatics, and with its growing popularity such applications will need to develop mechanisms to protect the privacy of the LLs, and/or BYs, for example (avoid misclosure - wrongly sharing content), when lifelog images are shared online. Hence designing privacy preserving mechanisms should be considered an integral part, while developing lifelogging platforms for the LLs. Our findings also demonstrated that the image contents (i.e. objects and known people in the image, activities captured and how these activities represent the LLs), context of the image (i.e. scenario where the image is captured, perceived sensitivity of the image, and location), and audience who can view it, are likely to influence the sharing decisions of the LLs. These findings underscore the need to develop techniques that could automatically recommend, whether a lifelog image is suitable for sharing online. Existing state-of-the-art computer vision techniques to detect the number of faces in the images [4], objects present in the image (object detection [5]), activities portrayed in the image (activity and pose detection [11]), could be applied to process the image contents. Context of an image could be analyzed using the location logs, indoor or outdoor classification [2], and then using visual classifiers [10]. Finally, the results of the content and context analysis can be combined to recommend a sharing decision to the LLs. These recommendations must be presented in an intuitive and light weight manner, so that the LLs interaction is neither constrained nor cumbersome because while valued, privacy is not the users' primary task. We do not claim that our study with LLs is rigorous, but contend that the results warrant further research to develop user friendly lifelogging applications.

\section{Future Work}

Our study was conducted with novice users (since lifelogging is still in its infancy) and the period of study was limited to avoid subject attrition. In the future, we aim to conduct the study over an extended period of time by recruiting the subjects well acquainted with the lifelogging technology, to further gain insights about 
lifelog sharing. Moreover, we plan to further enhance the protocol by making the subjects share their lifelog images in a private platform to better understand the phenomena of sharing lifelogs, especially the difficulties faced while sharing lifelog images from a collection formed over a substantial period of time, and types of images shared. The current study provides useful insights to develop experiment protocol to study lifelogging in the wild. The privacy implications associated with sharing lifelogs online will depend upon many factors, one of which is possible audience. First, we aim to list a number of scenarios where LLs will capture images, and the likely sharing groups in their online social circle. Then our goal is to understand the degree to which lifelogs captured in different scenarios are perceived as sensitive by the LLs, and LLs' sharing preferences for the lifelogs captured in different scenarios. The results are likely to help in recommending sharing decisions by considering both the image scenario and audience. This could be improved for a LL by developing a classifier, which will learn from the preferences made by the LL for images captured in different scenarios.

\section{References}

1. Ackerman, M.S. and Mainwaring, S.D., 2005. Privacy issues and human-computer interaction. Computer, 27(5), pp.19-26.

2. Byrne, D, et al., 2010. Everyday concept detection in visual lifelogs: validation, relationships and trends. Multimedia Tools and Applications, 49(1), pp.119-144.

3. Caprani, N., Gurrin, C. and O'Connor, N.E., 2012. Sharing as a motivation for lifelogging.

4. Doherty, A.R. and Smeaton, A.F., 2008. Combining face detection and novelty to identify important events in a visual lifelog. In Computer and Information Technology Workshops, 2008. IEEE 8th International Conference on (pp. 348-353). IEEE.

5. Doherty, A.R, et al., 2008, October. Combining image descriptors to effectively retrieve events from visual lifelogs. In Proceedings of the 1st ACM international conference on Multimedia information retrieval (pp. 10-17). ACM.

6. Gürses, S., Troncoso, C. and Diaz, C., 2011. Engineering privacy by design.Computers, Privacy \& Data Protection, 14, p.3.

7. Gurrin, C, et al., 2014. A privacy by design approach to lifelogging. Digital Enlightenment Yearbook 2014: Social Networks and Social Machines, Surveillance and Empowerment.

8. Hoyle, R, et al., 2014, September. Privacy behaviors of lifeloggers using wearable cameras. In Proceedings of the 2014 ACM Joint Conference on Pervasive and Ubiquitous Computing (pp. 571-582). ACM.

9. Hoyle, R, et al., 2015, April. Sensitive Lifelogs: A Privacy Analysis of Photos from Wearable Cameras. In Proceedings of the 33rd Annual ACM Conference on Human Factors in Computing Systems (pp. 1645-1648). ACM.

10. Oliva, A. and Torralba, A., 2001. Modelling the shape of the scene: A holistic representation of the spatial envelope. International journal of computer vision, 42(3), pp.145-175.

11. Pirsiavash, H. and Ramanan, D., 2012, June. Detecting activities of daily living in first-person camera views. In Computer Vision and Pattern Recognition, 2012 (pp. 2847-2854). IEEE.

12. Zhou, L.M. and Gurrin, C., 2012. A survey on life logging data capturing.SenseCam 2012. 